\title{
ETIOLOGY OF AUTISM AND PARENTS OF AUTISTIC CHILDREN
}

\author{
Elona Mano \\ PhD Candidate, European University of Tirana, kristinamano@yahoo.com
}

\begin{abstract}
There is an upward trend of rates in recent decades of autistic children. Since the Kanner's initial description of autism (1943) the increase in rate has led to dramatic claims. Several factors complicate the interpretation of the apparent increase, including changes in diagnostic practice, earlier diagnosis, increased awareness of the disorder, issues of study design and case ascertainment. A common source of confusion has been the tendency to mistake prevalence for incidence; prevalence refers to cases with the disorder at a specified time whereas incidence refers to the rate of new cases of the disorder within a period of time. The undiscovered cause of autism is the focus of many studies today. In order to cope with the stressful situation, it is very important for parents of autistic children to find some explanation for their child's developmental disorder. The pace of research has increased dramatically in recent years. A lot of studies have focused on bringing together work from diverse areas such as psychological and brain mechanisms and the social deficit of autism. Despite the collaborative studies on diagnosis, genetics, psychopharmacology, treatment and neuroimaging, however, much is left to do. This study involved mothers and fathers of children diagnosed with autism spectrum disorder. Three focus groups were formed. The interview was conducted in an informal and natural way where respondents were free to give their own views. Members of groups described feelings of sadness and anger, but they were eager to help their children. It seems that the etiology explanation from parents' point of view is connected with nature-nurture problem.
\end{abstract}

Keywords: Autism, Parents, Etiology, Focus group

\section{EPIDEMIOLOGY OF AUTISM}

Autism spectrum disorder (ASD) and autism are both general terms for a group of complex disorders of brain development. Based on DSM-VI-TR, (Diagnostic and Statistical Manual of Mental Disorder) in the spectrum is included diagnoses of autism, Asperger syndrome, pervasive developmental disorder not otherwise specified (PDD-NOS), Rett's syndrome and childhood disintegrative disorder. These disorders are characterized, in varying degrees, by difficulties in social interaction, verbal and nonverbal communication and repetitive behaviors.

Leo Kanner (1894-1981) was an Austrian psychiatrist and physician known for his work related to autism. In 1943, Kanner published a landmark paper, "Autistic Disturbances of Affective Contact" describing eleven 
children who were highly intelligent but displayed "a powerful desire for aloneness" and "an obsessive insistence on persistent sameness. Later he names their condition "early infantile autism." This is now known as autism.

Compared to Kanner's first steps there is an upward trend of rates in recent decades of autistic children. The rate for studies published between 1966 and 1991 was 4.4 cases per 10,000, while that for $1992-2001$ was 12.7 (Fombonne, 2003b). Based on the evidence reviewed, the median of prevalence estimates of autism spectrum disorders was 62/10 000. Elsabbagh, M, et al., (2012), but we still cannot talk about autism epidemic. The high rates of prevalence and diagnosis today are evidence that scientists are counting cases correctly. Grinker, R, R. (1997).

Several factors complicate this situation. There is a tendency to mistake prevalence for incidence. Prevalence refers to cases with the disorder at a specified time whereas incidence refers to the rate of new cases of the disorder within a period of time (usually a year). In general, epidemiological studies have focused on strictly defined autism, not including other conditions of the spectre. Volkmar, R. F., Lord C., Bailey, A., Schultz T. R., Klin A. (2004).

Changes in diagnostic practice is another factor. Twenty six years ago, participants in the studies would have been classified as meeting or not meeting DSM III-R (Diagnostic and Statistical Manual of Mental Disorder) without a standard method of clarifying the nature of differences between the groups. The diagnosis of autism and related conditions became increasingly standardized by 2000 . Identifying the defining deficits of autism became easier with DSM-IV and ICD-10 (The International Classification of Deseases) for most of autism-related categories, using instruments such as the Autism Diagnostic InterviewRevised and the Autism Diagnostic Observation Schedule. In 2013, based on DSM- V there were five major changes: 1.The new classification system eliminates the previously separate subcategories on the autism spectrum, including Asperger syndrome, PDD-NOS, childhood disintegrative disorder and autistic disorder. These subcategories will be folded into the broad term autism spectrum disorder (ASD). 2. Instead of three domains of autism symptoms (social impairment, language/communication impairment and repetitive/restricted behaviors), two categories will be used: social communication impairment and restricted interests/repetitive behaviors. Under the DSM-IV, a person qualified for an ASD diagnosis by exhibiting at least six of twelve deficits in social interaction, communication or repetitive behaviors. Under the DSM-5, diagnosis will require a person to exhibit three deficits in social communication and at least two symptoms in the category of restricted range of activities/repetitive behaviors. Within the second category, a new symptom will be included: hyper- or hypo-reactivity to sensory input or unusual interests in sensory aspects of the environment.3.Symptoms can currently be present, or reported in past history.4.In addition to the diagnosis, each person evaluated will also be described in terms of any known genetic cause (e.g. fragile X syndrome, Rett syndrome), level of language and intellectual disability and presence of medical conditions such as seizures, anxiety, depression, and/or gastrointestinal (GI) problems.5.The work group added a new category called Social Communication Disorder (SCD). This will allow for a diagnosis of disabilities in social communication without the presence of repetitive behavior. Autism speaks (2015).

Compared to 20 years ago there is an increase awareness of the disorder. Parents awareness led to earlier diagnosis. The early onset of autism and the evidence showing the considerable benefits of early intervention is a good sign for parents of autistic children. Volkmar R.V. et al.

To live with an autistic child is a very demanding and stressful situation. Tupja, E. (2009). Caring for a child with autism can be a twenty-four hour job throughout the child's entire lifetime. Dumas et al, 1991 found that parents of children with autism and behavior disorders reported significantly higher levels of parenting stress than parents of children with Down Syndrome or with normal development. A child with autism is a large stressor on the family because of the ambiguity of diagnosis, the severity and duration of the disorder, and problems with the child's lack of adherence to social norms (Bristol, 1984).

\section{ETIOLOGY OF AUTISM}

Kanner's initial report (1943) of the syndrome of early infantile autism based on association between autism and social status of the parents. Parents were part of professions like writers, physicians, psychologists, pathologists, lawyers, psychiatrists and chemists.

"There is one other very interesting common dominator in the backgrounds of these children. They all come of highly intelligent families."

Later studies fail to find any association. (Fombonne, 2003).

Based on Kanner's finding, some researchers relate autism etiology with 'frigid' mothers. 
"One other fact stands out prominently. In the whole group, there were very few warmhearted fathers and mothers... The question arises whether or to what extent this fact has contributed to the condition of the child."

In the same paper he admits that autism is an innate disorder.

"We must assume that these children have came into the world with innate inability to form the usual, biologically provided affective contact with people." Kanner L. (1943)

Kanner's attitude is not such an ambiguity example, because on one side he is positive that autism is an innate disorder and on the other side he is still wondering, that means he is not sure about 'Frigid' mothers etiology.

Other potential risk for autism has been immigrant status or even the race of parents, but the evidences to support these hypotheses were weak. Reports of 'autbreaks' of cases of autism attract attention of the lay media in USA and UK, but even here there were no convincing evidences. Ten years ago a topic of debate remained the notion that autism might be caused by measles vaccination MMR and mercury based vaccines. As a result rates of measles vaccination fell in the UK. Evans et al. (2001). In 2004, a comprehensive review by the Institute of Medicine concluded that the evidence favors rejection of possible causal associations between each of these vaccine types and autism.

"Beginning at birth, an infant is exposed to hundreds of viruses and other antigens, and it has been estimated that an infant theoretically could respond to thousands of vaccines at once. The possibility that immunologic stimulation from vaccines during the first 1-2 years of life could be related to the development of $A S D$ is not well supported by the known neurobiology of $A S D$, which tends to be genetically determined with origins in prenatal development. (De Stefano F, Price. S. C., Weintraub S. E. 2004)"

Because of variability in syndrome manifestation and lack of specific factors the researchers have been focused on core psychological markers that can help in genetic and neuroimaging research. Since 1990 there have been studied a few dominant psychological models of autism based on 'theory of mind', that is related with difficulty of autistic people to understand the other people, in base of their attitudes, emotions or other's point of view.

"The theory of mind hypothesis... defines the social dysfunction in autism as the result of disruptions in processes leading to the acquisition of the capacity for conceiving of other people 's and one 's own mind."

(Baron- Cohen, 1995; Volkmar R. F. et al 2004)

Because the autism can be identified within the first two years of life and by this age usually children develop social interactions there is a need for researchers to set forth a psychological model which should focus on skills and processes that both emerge early and that could eventually lead to deficits in theory of mind skills.

In the past decade functional neuroimaging studies have test some psychological hypotheses. One important finding was the discover of generalized hypoactivation of an entire social processing brain network in individuals with autism. Even though this deserves further investigation, this finding could be a sign of pervasive lack of social interest, engagement, motivation, or reactivity. Volkmar R. F. et al. (2004).

\section{METHOD}

This study is guided by social ecological systems theory (Bronfenbrenner, 1979), who suggests that the ecological system comprises a number of subsystems: the microsystem, mesosystem, exosystem and macrosystem. To understand children`s development, we should take into account not only the children but even the microsystem to which the child has direct contact. A microsystem typically includes family, peers, or caregivers. Relationships in a microsystem are bi-directional. In other words this theoretical framework suggests that parents-children relationships are likely to influence parents 'point of view. This study focuses on parents of autistic children reports and is guided by the following question: What is the cause of autism from parents' point of view.

\subsection{Participants}

The sample for the current study includes mothers, fathers and grandparents of autistic children diagnosed with Autism Spectrum Disorder (Asperger`s Disorder, Autistic Disorder, Rett's syndrome, Childhood Disintegrative Disorder, Pervasive Developmental Disorder, not otherwise specified) based on DSM-IV-TR (Diagnostic and Statistical Manual of Mental Disabilities). Parents are members of MEDPAK (Protect the Rights of Parents of Persons with Disabilities), a non-governmental organisation in Albania. 


\subsection{Procedure}

Information was collected from mothers, fathers and grandparents as part of three different focus groups. The first group was compound by ten mothers of children with autism spectrum disorder. The second group was that of five fathers of autistic children. The third group included seven grandmothers of autistic children. The interviews were conducted in different days. The moderator gave an overview of the topic, made them sure that everyone point of view would be appreciated. They were made aware they were free to withdraw from the study at any time for any reason. After the moderator created a warm and friendly environment, confidentiality was ensured to all participants.

It was used a play back equipment. An assistant moderator took control over handles logistics and took careful notes. During the process moderator used pauses and probes. The questions used were openended questions, such as: how did you realise that your child was part of the spectrum disorder? How did you feel about it? What causes your child's condition. The interviews lasted about 60 minutes.

\subsection{Systematic Analysis Process}

It was used the textual analysis, which includes a wide variety of things that can be analysed. The process of qualitative analysis aims to bring meaning to a situation rather than the search for truth focused on by quantitative research. In order to minimize the potential bias introduced in analysing and interpreting focus group data, Krueger \& Casey (2000) point out that the analysis should be systematic, sequential, verifiable, and continuous.

The first step was indexing and charting as a form to manage the data. It was used a computer-based approach for cutting, pasting, sorting, arranging and rearranging data through comparing and contrasting the relevant information. It was numbered each line of each transcript and two hard copies of each transcript were made; one to cut up and one that stays intact for later reference. Later the transcripts were printed on different colored paper to identify the groups. Then it was arranged the working transcript in a reasonable order, i.e. categories of participants: age, male, female. This arrangement helped the moderator to be alert to changes that maybe occurring from one group to another. At the end it was written on each page one of the focus group questions to be analyzed. Because the author had several categories of groups, lines were drawn to divide the paper into sections and then group comments within these sections. For example, on one part of the page the author placed comments from mothers focus groups, in another place there were comments from fathers focus groups, and in a third place there were comments from grandmothers focus groups. Later responses to the same question from all focus groups were read. The author cut out relevant quotes and taped them to the appropriate place on the large sheet of paper. The quality of quotes varied. In mother's group all the quotes were used, but in grandmothers quotes not all of them were used. The unused quotes were left aside for later consideration. At the end of this process an overview was prepared integrating paragraph that described responses to that question. When this was finished, the author went to the next question, and so on.

For interpretation of coded data Krueger (1994) provides seven established criteria: words; context; internal consistency; frequency and extensiveness of comments; specificity of comments; intensity of comments; big ideas. First of all, the moderator thought about the actual words used by the participants and the meanings of those words. The analyst needed to determine the degree of similarity between the responses. Later the analyst examined the context by finding the stimulus that triggered participants' responses and interpreted the comment in light of the environment. Sometimes there was a shift in opinion and in order to discover the internal consistency, the researcher traced the flow of the conversation. The frequency relates to consideration of how often a comment is made. There were topics of special interest to participants. The conversation was not video- taped so the intensity was difficult to be spotted. Intensity is communicated by the voice tone, speed or emphases on different words. Based on specificity, responses that were specific and based on the first person experiences were given greater attention. Another thing to be considered by the analyst was 'big ideas'. Krueger (1994) suggests taking a break for a few days at this stage in order to refocus on the big picture.

It was very important for all participants to find a possible cause of their child's autism. It was interesting that many parents suggested that some type of birth trauma caused their child's autism, specially fathers of autistic children. $30 \%$ of all participants believe that the MMR vaccination caused a reaction that led to autism. A lot of mothers suggested that there was a genetic cause for their child condition. There were even opinions that autism is caused by some kind of curse or bad luck or even by something the child ate. This was found in grandmothers group and mothers group. Members of groups described feelings of sadness and anger, but they were eager to help their children, first of all by trying to find answers to what had happened to their children. 
Ms.6, mother of an autistic boy says:

"... why me?. Have I done something wrong in my life and somebody cursed me?"

Ms.3, mother of two, one of which is autistic says:

"...It is the vaccination. I know. But how could they...?"

One of the most touching issues for parents of autistic children is the future of their children. They understand that autism is un curable and this is why they are scared of tomorrow, when they will not be alive.

\section{DISCUSSION}

The purpose of this study was to perceive parent's opinion concerning the etiology of autism. Based on the text analyses it seems that the cause of autism is that of nature/nurture or environment versus heredity. This debate is one of the most enduring and controversial not only in psychology, but even in religious, philosophical, political and scientific context. First of all the debate was about nature or nurture. It relates to which is more important regarding the nature-nurture debate.

By 1960 biologists began to see the issue from another point of view, the interaction between heredity and environment Dunbar (2008). Later the debate took the form of 'How much', trying to quantify the contributions of genetic and environmental factors. This is related with behaviour genetics, which attempts to establish the extent to which individual differences are due to differences in people's genetic makeup. Related to this question was another question 'How do they interact?' This question is about the ways in which heredity and environment influence each other. Gross R. 2009

Today the main issue in this debate is how we define 'the environment'. What 'the environment is'. Gross R. 2009 gives us the clue to understand this notion based on psychological context:

"...we normally think of all those influences, or potential sources of influence, that lie outside the individual's body, in the form of other people, opportunities for intellectual stimulation and social interaction, as well as physical circumstances of the individual's life ('environs'= 'surroundings')."

For Watson the environment was something existing independently of the individual.

"The behaviourists believe that there is nothing from within to develop. If you start with the right number of fingers and toes, eyes, and a few elementary movements that are present at birth, you do not need anything else in the way of raw material to make a man, be that man genius, a cultured gentleman, a rowdy or a thug." (Watson, 1925/26, in Soyland, 1994, in Gross R. 2009)

Another view of what the environment is, to see how people create an environment. The pre-natal, biological environment of a baby is in risk by many factors such as: mother's diet, maternal stress, X-rays and other radiation, accidents, toxic agents, drugs, or maternal diseases. Rose (2005) claims that the environment exists in multiple levels. In a piece of DNA, 'the environment' is all the rest of the DNA in the genome, plus the cellular metabolic system that surrounds it, proteins, enzymes, ions, water and so on. For a cell in a multicellular organism, the environment is the internal milieu in which it is embedded, signalling molecules, bloodstream and cellular fluids. Heredity refers only to the particular set of chromosomes and genes that combine at the moment of fertilisation; anything that occurs from that moment on is environmental. (Kirby and Radford, 1976; Rose, 2005). Seen in this way the interaction gene-environment can arise before the birth. Been in different environmental conditions an individual is influenced in different ways on genetic inheritance. (McGurk, 1975 ).

\section{ACKNOWLEDGEMENT}

This study could not have been completed without the support given by my superviser and all of the families involved in this study, either as participants in the sample or as collaborators in the research design. Thanks are given to the local authority and IT specialist for helping me during the research.

\section{REFERENCE LIST}

Bronfenbrenner, U, (1979), The ecology of human development, Boston: Harvard University.

Bristol, M. M. (1984). Family resources and successful adaptation to autistic children. In E. Schopler \& G. B. Mesibov (Eds), The effects of autism on the family (pp. 289-310). New York: Plenum Press.

Dumas, J.E., Wolf, L.C., Fisman, S.N., \& Culligan, A. (1991). Parenting stress, child behavior problems, and dysphoria in parents of children with autism, Down syndrome, behavior disorders, and normal development. Exceptionality, 2, 97-110. 
Dunbar, R. (2008) Taking evolutionary psychology seriously. The psychologist, 21(4), 304-6.

De Stefano F., Price S.C., Weintraub S. E. Journal of Pediatrics 2004. www.jpeds.com

Evans M et al. The British Journal of General Practice.2001 51 (472):904-910

Gross R. 2009. Themes, Issues and debates in Psychology. Hodder Education. London.UK.

Grinker, R. R. (2007), Unstrange Minds, Basic Books

Krueger RA \& Casey MA (2000) Focus Groups: A Practical Guide for Applied Research, 3rd ed. Thousand Oaks, CA: Sage publications

Krueger RA (1994) Focus Groups: A Practical Guide for Applied Research. Thousand Oaks, CA: Sage Publications.

Kirby, R. and Radford, J. (1976) Individual Differences. London: Methuen

Kanner, L. (1943) Autistic Disturbances of affective contact. Nervous Child 2,217-250.

McGurk, H. (1975) Growing and Changing. London: Methuen.

Rose, S. (2005) The 21st Century Brain: Explaining, Mending and Manipulating the Mind. London: Vintage Books.

Tupja, E. (1009). Kur dhimbja premton dashuri. OMBRA GVG, pg.29.

Fombonne E. The prevalence of autism, Journal of the American Medical Association. 2003b; 289:1-3. [PubMed]

Volkmar, R. F., Lord C., Bailey, A., Schultz T. R., Klin A. (2004) Journal of Child Psychology and Psychiatry $45,1,(135-170)$

Elsabbagh, M. et al,. 2012 Global Prevalence of Autism and other PDD. (October 2015) pubMed http://www.ncbi.nlm.nih.gov/pubmed/22495912

Autism Speaks. www.autismspeak.org 2015 